\title{
OPERATORS SATISFYING CERTAIN GROWTH CONDITIONS
}

\author{
S. M. PATEL AND B. C. GUPTA
}

ABSTRACT. Let $T$ be an operator on a complex Hilbert space $H$. Some growth conditions on operator radius of the resolvent of $T$ are studied. Moreover, it is shown that the conjecture, due to V. Istrățescu, that for operators $T$ satisfying growth condition $\left(G_{1}\right)$

$$
\sup _{\|x\|=1}\left\{\|T x\|^{2}-|(T x, x)|^{2}\right\}=R_{T}^{2},
$$

where $R_{T}$ is the radius of the smallest circular disk containing the spectrum $\sigma(T)$, turns out to be false.

Let $T$ be an operator on a complex Hilbert space $H$. Let $\pi(T), \pi_{0}(T)$ and $W(T)$ denote the approximate point spectrum, point spectrum and the numerical range of $T$. We write $r(T)$ and $|W(T)|$ for the spectral radius and the numerical radius of $T$, Let Bdry $\sigma(T)$ and Conv $\sigma(T)$ denote the boundary and the convex hull of $\sigma(T)$.

An operator $T$ is called transloid if $T+z I$ is normaloid, that is, if $r(T+z I)=\|T+z I\|$ for all complex numbers $z$. If $\left\|(T-z I)^{-1}\right\|=$ $1 / d(z, \sigma(T))$, where $d(z, \sigma(T))$ denotes the distance of $\sigma(T)$ from the point $z$ (equivalently if $(T-z I)^{-1}$ is normaloid for all $z \notin \sigma(T)$ ), then $T$ is said to satisfy the growth condition $\left(G_{1}\right)$ or $T$ is called an operator with $\left(G_{1}\right)$ property.

Let $C_{\rho}(\rho>0)$ be the class of all operators with unitary $\rho$-dilation in the sense of [6]. According to Holbrook [3], an operator radius of $T$ is defined by

$$
w_{\rho}(T)=\inf \left\{\alpha: \alpha>0 \text { and } \alpha^{-1} T \in C_{\rho}\right\} .
$$

In particular, $w_{1}(T)=\|T\|$ and $w_{2}(T)=|W(T)|$. For further properties of operator radii, we refer to [3]. An operator $T$ is called $\rho$-oid if $w_{\rho}(T)=$ $r(T)$. Clearly, 1-oid and 2-oid operators are, respectively, the normaloid and spectraloid operators. According to [9], $T$ is called an operator of class $M_{\rho}(\rho \geq 1)$ if $\left.w f(T-z I)^{-1}\right]=1 / d(z, \sigma(T))$; equivalently if $(T-z I)^{-1}$ is $\rho$-oid for all $z \notin \sigma(T)$. Clearly $M_{1}$ consists of all operators with the $\left(G_{1}\right)$ property, and since $M_{\rho} \subseteq M_{\rho^{\prime}}$ for $\rho<\rho^{\prime}$, it follows, in particular, that $M_{1} \subseteq$

Received by the editors October 29, 1973 and, in revised form, December 12, 1974.

AMS (MOS) subject classifications (1970). Primary 47A20, 47A10.

Key words and phrases. Hilbert space operators, podilation, operator radii, transloid operators, selfadjoint and unitary operators, operators satisfying the growth condition $\left(G_{1}\right)$. 
$M_{\rho}$ for all $\rho \geq 1$. The fact that operators of class $M_{\rho}$ are convexoid follows immediately from the following criterion for convexoidity [9] which is an improved form of the well-known criterion due to Orland [8]: An operator $T$ is convexoid if and only if $w_{\rho}\left[(T-z I)^{-1}\right] \leq 1 / d(z$, conv $\sigma(T))$ for all $z \notin \operatorname{conv} \sigma(T)$.

Recently, Lin [13, Theorem 5] has obtained the following improvement of Nieminen's result [7, Theorem 1]: $T$ is selfadjoint if and only if $w_{\rho}\left[(T-i \xi)^{-1}\right] \leq 1 /|\xi|$ for all real $\xi \neq 0,1 \leq \rho \leq 2$. Our first result shows that it is true even for $\rho>2$.

Theorem 1. If $T$ is an operator such that the imaginary axis, with the possible exception of the origin, belongs to the resolvent set of $T$ and $w_{\rho}\left[(T-i \xi)^{-1}\right] \leq 1 /|\xi|$ for every real $\xi \neq 0$, then $T$ is selfadjoint.

Proof. Since the given condition implies

$$
w_{\rho}\left[\left(I+i \xi^{-1} T\right)^{-1}\right]=|\xi| w_{\rho}\left[(T-i \xi)^{-1}\right] \leq 1, \quad\left(I+i \xi^{-1} T\right)^{-1} \in C_{\rho} .
$$

Using [6, Theorem I.11.1] with $z=1$ we obtain

$$
(\rho-2)\left\|\left(I-\left(I+i \xi^{-1} T\right)^{-1}\right) x\right\|^{2}+2 \operatorname{Re}\left(\left(I-\left(I+i \xi^{-1} T\right)^{-1}\right) x, x\right) \geq 0
$$

for all $x \in H$, that is,

$$
(\rho-2)\left\|i \xi^{-1} T\left(I+i \xi^{-1} T\right)^{-1} x\right\|^{2}+2 \operatorname{Re}\left(i \xi^{-1} T\left(I+i \xi^{-1} T\right)^{-1} x, x\right) \geq 0 .
$$

Writing $\left(I+i \xi^{-1} T\right)^{-1} x=y$, we get

$$
(\rho-2)\left\|i \xi^{-1} T y\right\|^{2}+2 \operatorname{Re}\left(i \xi^{-1} T y,\left(I+i \xi^{-1} T\right) y\right) \geq 0
$$

for all $y \in H$, that is

$$
(\rho-2)\left|\xi^{-2}\right|\|T y\|^{2}-2 \xi^{-1} \operatorname{Im}(T y, y)+2\left|\xi^{-2}\right|\|T y\|^{2} \geq 0,
$$

or

$$
\left(\rho /|\xi|^{2}\right)\|T y\|^{2} \geq 2 \xi^{-1} \operatorname{Im}(T y, y)
$$

or

$$
\rho\|T y\|^{2} \geq 2|\xi|^{2} \xi^{-1} \operatorname{Im}(T y, y) .
$$

Now let $\xi=n \operatorname{Im}(T y, y)$; we have

$$
2 n|\operatorname{Im}(T y, y)|^{2} \leq \rho\|T y\|^{2} .
$$

Taking limit as $n \rightarrow \infty$, we get $\operatorname{Im}(T y, y)=0$, or $(T y, y)=(y, T y)$ for every $y \in H$. Therefore $T$ is selfadjoint. This completes the proof.

Next, we would like to ask whether the result of Donoghue [2] can be 
improved along similar lines. More explicitly: Is $T$ unitary if $w_{a}\left(T^{-1}\right) \leq$ 1 and $w_{\rho}\left[(T-z)^{-1}\right] \leq 1 /(|z|-1),|z|>1$ ? The following theorem answers the question affirmatively.

Theorem 2. Let $T$ be an operator such that:

(i) $\sigma(T) \subseteq C$, where $C$ is the unit circle;

(ii) $w_{\alpha}\left(T^{-1}\right) \leq 1, a \geq 1$; and

(iii) $w_{\rho}\left[(T-z)^{-1}\right] \leq 1 /(|z|-1), 1<|z|<\delta<(\rho-1) /(\rho-2), \rho>2$.

Then $T$ is unitary.

Proof. First we observe that conditions (ii) and (iii) are equivalent to $T^{-1} \in C_{a}$ and $(|z|-1)(T-z)^{-1} \in C_{\rho}$ [3, Theorem 3.1], respectively. By Remark 3 of [6, Proposition I.11.2], the latter condition yields

$$
\begin{aligned}
&\left\|\left[(T-z)^{-1}-\mu /(|z|-1)\right]^{-1}\right\| \leq(|z|-1) /(|\mu|-1) \\
& 1<|\mu|<(\rho-1) /(\rho-2) .
\end{aligned}
$$

Since $|\mu| /(|z|-1)>(|\mu|-1) /(|z|-1)$, Lemma 1 of [12] gives

$$
\begin{aligned}
&\left\|[T-z-(|z|-1) \bar{\mu} /(2|\mu|-1)]^{-1}\right\| \leq(2|\mu|-1) /(|\mu|-1)(|z|-1), \\
& 1<|\mu|<(\rho-1) /(\rho-2) .
\end{aligned}
$$

Setting $z=-\bar{\mu}$, we obtain

$$
\left\|(T-u)^{-1}\right\| \leq 1 /(|u|-1), \quad 1<|u|<k,
$$

where $u=-|\mu| \bar{\mu} /(2|\mu|-1)$ and $k$ is some constant. Therefore $T \in C_{\beta}$ for some $\beta>2$. Since $T^{-1} \in C_{\alpha}$, by Corollary 4 of [12] it follows that $T$ is unitary. The proof is complete.

In particular, the following corollary includes Donoghue's result [2].

Corollary 1. Let $T$ be an operator such that

(i) $\sigma(T) \subseteq C$,

(ii) $w_{a}\left(T^{-1}\right) \leq 1, \alpha \geq 1$, and

(iii) $w_{\rho}\left[(T-z)^{-1}\right] \leq 1 /(|z|-1), 1<|z|<\delta, 1 \leq \rho \leq 2$.

Then $T$ is unitary.

Proof. Choose $\rho^{\prime}$ such that $\rho \leq 2<\rho^{\prime}<(2 \delta-1) /(\delta-1)$. Then it follows from (iii) that $(|z|-1)(T-z)^{-1} \in C_{\rho} \subseteq C_{\rho^{\prime}}, 1<|z|<\delta<\left(\rho^{\prime}-1\right) /\left(\rho^{\prime}-2\right)$. Hence, the result follows from Theorem 2.

Corollary 2. If $T \in M_{\rho}$ and $\sigma(T) \subseteq C$, then $T$ is unitary.

Proof. Since $T \in M_{\rho}$,

$$
w_{\rho}\left[(T-z)^{-1}\right] \leq 1 / d(z, \sigma(T)) \leq 1 /(|| z|-1|)
$$

for all $z \notin \sigma(T)$. In particular, we have $w_{\rho}\left(T^{-1}\right) \leq 1$ and $w_{\rho}\left[(T-z)^{-1}\right] \leq$ $1 /(|z|-1)$ for $|z|>1$. Therefore the result follows from Theorem 2 and Corollary 1 . 
Our next theorem includes the following result implicitly proved by T. Saito [10, Theor em 1].

Theorem A. Let $T$ be an operator satisfying the growth condition $\left(\mathrm{G}_{1}\right)$ and let $a_{0}$ be a point in $\sigma(T)$. If there exist sequences $\left\{z_{n}\right\}$ and $\left\{r_{n}\right\}$ such that

(i) $\left\{a:\left|\alpha-z_{n}\right|<r_{n}\right\}$ is contained in the complement of $\sigma(T)$,

(ii) $z_{n} \rightarrow \alpha_{0}$ and $\left|z_{n}-a_{0}\right| / r_{n} \rightarrow 1$, then

$$
E\left(T-\alpha_{0} I\right)=E\left(T^{*}-\bar{\alpha}_{0} I\right)
$$

where

$$
E\left(T-a_{0} I\right)=\left\{\left\{x_{n}\right\}: x_{n} \in H,\left\|x_{n}\right\|=1 \text { and }\left\|\left(T-\alpha_{0} I\right) x_{n}\right\| \rightarrow 0\right\} .
$$

Theorem 3. Let $T$ be an operator of class $M_{\rho}$. If $\alpha_{0}$ is a point of $\sigma(T)$ satisfying the conditions of Theorem $A$, then $E\left(T-a_{0} I\right)=E\left(T^{*}-\bar{a}_{0} I\right)$.

Proof. Changing the Hilbert space suitably and by a faithful *-representation $T \rightarrow T^{0}$, one can suppose $\pi(T)=\pi_{0}(T)$ and thereupon, Bdry $\sigma(T) \subseteq$ $\pi(T)=\pi_{0}(T)$ [1]. Since $T \geq 0$ if and only if $T^{0} \geq 0$, it is easily seen that $T \in C_{\rho}$ if and only if $T^{0} \in C_{\rho}$. Consequently, $w_{\rho}(T)=u_{\rho}\left(T^{0}\right)$. To prove the assertion, it will suffice to show that $\operatorname{ker}\left(T-a_{0} l\right)=\operatorname{ker}\left(T^{*}-\bar{\alpha}_{0} l\right)$.

Since $T-z I \in M_{\rho}$ whenever $T \in M_{\rho}$, we can assume that $a_{0}=0$. Then $z_{n} \rightarrow 0$ and $\left|z_{n}\right| / r_{n} \rightarrow 1$. Let $a_{n}=\left|z_{n}\right|-r_{n}$. Clearly, $\alpha_{n} / r_{n} \rightarrow 0$. Let $a_{n}$ $=e^{i \arg z_{n}}$ and $T_{n}=\left(a_{n} a_{n}-z_{n}\right)\left(T-z_{n}\right)^{-1}$. Then, by hypothesis,

$$
\begin{aligned}
w_{\rho}\left(T_{n}\right) & =\left|a_{n} \alpha_{n}-z_{n}\right| w_{\rho}\left[\left(T-z_{n}\right)^{-1}\right] \\
& \leq\left|a_{n} \alpha_{n}-z_{n}\right| / d\left(z_{n}, \sigma(T)\right)=\left|a_{n}\right| r_{n} / d\left(z_{n}, \sigma(T)\right) \leq 1 .
\end{aligned}
$$

This shows that $T_{n} \in C_{\rho}[3$, Theorem 3.1]. Now we assert that

(a) $T x=0 \Leftrightarrow T_{n} x \rightarrow x_{\text {, and }}$

(b) $T^{*} x=0 \Leftrightarrow T_{n}^{*} x \rightarrow x$.

By symmetry, it suffices to prove (a). Assume that $T x=0$. Then

$$
\begin{aligned}
\left\|T_{n} x-x\right\| & \leq \rho\left\|x-T_{n}^{-1} x\right\|=\rho\left\|x+\left(1 / a_{n}{ }_{n}\right)\left(T-z_{n}\right) x\right\| \\
& =\rho\left\|\left(I-\left|z_{n}\right| / r_{n}\right) x\right\| \rightarrow 0 .
\end{aligned}
$$

Thus $T_{n} x \rightarrow x$. On the other hand, if $T_{n} x \rightarrow x$, then

$$
\begin{aligned}
\left\|\left(T-a_{n} \alpha_{n}\right) x\right\| & =\left\|\left[\left(T-z_{n}\right)-\left(a_{n} \alpha_{n}-z_{n}\right)\right] x\right\|=\left\|\left(T-z_{n}\right)\left(I-T_{n}\right) x\right\| \\
& \leq\left(\|T\|+\sup _{n}\left|z_{n}\right|\right)\left\|T_{n} x-x\right\| \rightarrow 0 .
\end{aligned}
$$

Therefore, 


$$
\|T x\|=\lim \left|a_{n}\left\|\alpha_{n} \mid\right\| x\left\|\leq\left(\sup r_{n}\right)\left(\lim \left(\left|\alpha_{n}\right| / r_{n}\right)\right)\right\| x \|=0 .\right.
$$

Thus $T x=0$. Now to complete the proof it is enough to show that $T_{n} x \rightarrow$ $x \Leftrightarrow T_{n}^{*} x \rightarrow x_{0}$ Since $T_{n} \in C_{\rho}$, it follows from [6, Theorem I.11.1] that

$$
(\rho-2)\left\|\left(I-T_{n}\right) v\right\|^{2}+2 \operatorname{Re}\left(\left(I-T_{n}\right) v, v\right) \geq 0, \quad v \in H .
$$

Thus $\operatorname{Re} S_{n} \geq 0$ where $S_{n}=(\rho-2)\left(I-T_{n}\right)^{*}\left(I-T_{n}\right)+2\left(I-T_{n}\right)$. Therefore we have

$$
\left|\left(\left(\operatorname{Re} S_{n}\right) x, y\right)\right|^{2} \leq\left(\left(\operatorname{Re} S_{n}\right) x, x\right)\left(\left(\operatorname{Re} S_{n}\right) y, y\right), \quad x, y \in H .
$$

Since $T_{n} \in C_{\rho}$ and, hence, $\left\|T_{n}\right\| \leq \rho$, it follows that the sequence $\left\{S_{n}\right\}$ is bounded; thus the last inequality reduces to

$$
\left|\left(\left(\operatorname{Re} S_{n}\right) x, y\right)\right|^{2} \leq k_{1}\|y\|^{2} \operatorname{Re}\left(S_{n} x, x\right), \quad x, y \in H,
$$

for some constant $k_{1}$.

Now if $T_{n} x \rightarrow x$, then $S_{n} x \rightarrow 0$. This, together with the above inequality, implies that $\left(\operatorname{Re} S_{n}\right) x \rightarrow 0$, and so $S_{n}^{*} x \rightarrow 0$, or $T_{n}^{*} x \rightarrow x$. A similar argument proves that $T_{n}^{*} x \rightarrow x \Rightarrow T_{n} x \rightarrow x$. This completes the proof.

V. Istrățescu [4, Theorem 2] has proved that if $T$ is transloid, then

$$
\sup _{\|x\|=1}\left\{\|T x\|^{2}-|(T x, x)|^{2}\right\}=R_{T}^{2}
$$

where $R_{T}$ is the radius of the smallest circular disk containing $\sigma(T)$. Moreover, he has conjectured that $(*)$ holds if $T$ is convexoid or more particularly an operator with $\left(G_{1}\right)$ property. Recently Sheth [11] has disproved the first part of the conjecture. Here we prove the falsity of the second part of the conjecture.

Theorem 4. There exists an operator satisfying the growth condition $\left(G_{1}\right)$ for which $(*)$ is not true.

Proof. Consider the operator $A=\left[\begin{array}{ll}0 & 1 \\ 0 & 0\end{array}\right]$ on the two-dimensional Hilbert space $H_{1}$. Let $B$ be a normal operator on an infinite-dimensional Hilbert space $H_{2}$ such that $\sigma(B)=W(A)$. Write $T=A \oplus B$. Then $T$ satisfies the growth condition $\left(\mathrm{G}_{1}\right)[5$, Theorem 1.2]. Let $x=(0,1) \oplus 0$. Clearly, $(T x, x)=0$ and $\|T x\|=1$. Therefore

$$
\sup _{\|x\|=1}\left\{\|T x\|^{2}-|(T x, x)|^{2}\right\} \geq 1 .
$$

Since $\sigma(T)=W(A)$ is the disk with the center at the origin and radius $1 / 2$, it follows that $R_{T}=1 / 2$. Thus

$$
\sup _{\|x\|=1}\left\{\|T x\|^{2}-|(T x, x)|^{2}\right\}>R_{T}^{2} .
$$

This proves the result. 
The authors express their sincere thanks to Dr. P. B. Ramanujan for his kind help and useful discussions during the preparation of this paper. Also they are grateful to the referee for his valuable suggestions to improve the original form of this paper.

\section{REFERENCES}

1. S. K. Berberian, Approximate proper vectors, Proc. Amer. Math. Soc. 13 (1962), 111-114. MR 24 \#A3516.

2. W. F. Donoghue, Jr., On a problem of Nieminen, Inst. Hautes Études Sci. Publ. Math. No. 16 (1963), 31-33. MR 27 \#2864.

3. J. A. R. Holbrook, On the power bounded operators of $S z=N a g y$ and Foias, Acta Sci. Math. (Szeged) 29 (1968), 299-310. MR 39 \#810.

4. V. Istrăteșcu, On a class of normaloid operators, Math. Z. 124 (1972), 199-202. MR 45 \#944.

5. G. R. Luecke, Topological properties of paranormal operators on Hilbert space, Trans. Amer. Math. Soc. 172 (1972), 35-43. MR 46 \#7951.

6. B. Sz.-Nagy and C. Foias,, Analyse harmonique des opérateurs de l'espace de Hilbert, Masson, Paris; Akad. Kiadó, Budapest, 1967; English rev. transl., NorthHolland, Amsterdam; American Elsevier, New York; Akad. Kiadó, Budapest, 1970. MR 37 \#778; 43 \#947.

7. T. Nieminen, A condition for the self-adjointness of a linear operator, Ann. Acad. Sci. Fenn. Ser. A I No. 316 (1962). MR 25 \#2452.

8. G. H. Orland, On a class of operators, Proc. Amer. Math. Soc. 15 (1964), 75-79. MR $28 \# 480$.

9. S. M. Patel, On some classes of operators associated with operator radii of Holbrook, Thirty-Ninth Annual Conf. of Indian Math. Soc., Jadavpur University, Jadavpur, 1973.

10. T. Saitô, A theorem on boundary spectra, Acta Sci. Math. (Szeged) 33 (1972), 101-104. MR $46 \# 4240$.

11. I. H. Sheth, On a conjecture of Is trătescu, J. Indian Math. Soc. (to appear).

12. J. G. Stampfli, A local spectral theory for operators, J. Functional Analysis 4 (1969), 1-10. MR 39 \#4698.

13. C.-S. Lin, On a family of generalized numerical ranges, Canad. J. Math. 16 (1974), 678-685.

FACULTY OF MATHEMA TICS, UNIVERSITY OF DELHI, DELHI-7, 110007 INDIA

DEPARTMENT OF MATHEMATICS, SARDAR PATEL UNIVERSITY, VALLABH VIDY ANAG AR-388120, GUJ AR AT, INDIA 